\title{
NO NEUTRON STAR COMPANION TO THE LOWEST MASS SDSS WHITE DWARF
}

\author{
Marcel A. Agüeros ${ }^{1,8}$, Craig Heinke ${ }^{2}$, Fernando Camilo ${ }^{1}$, Mukremin Kilic ${ }^{3,9}$, Scott F. Anderson ${ }^{4}$, Paulo Freire $^{5}$, \\ Scot J. Kleinman ${ }^{6}$, James W. Liebert $^{7}$, and Nicole M. Silvestri ${ }^{4}$ \\ ${ }^{1}$ Columbia Astrophysics Laboratory, Columbia University, New York, NY 10027, USA; marcel@astro.columbia.edu \\ ${ }^{2}$ Department of Physics, University of Alberta, Edmonton, AB T6G 2G7, Canada \\ ${ }^{3}$ Harvard-Smithsonian Center for Astrophysics, Cambridge, MA 02138, USA \\ ${ }^{4}$ Department of Astronomy, University of Washington, Seattle, WA 98195, USA \\ 5 Arecibo Observatory, Arecibo, PR 00612, USA \\ ${ }^{6}$ Gemini Observatory, Northern Operations Center, Hilo, HI 96720, USA \\ ${ }^{7}$ Steward Observatory, University of Arizona, Tucson, AZ 85721, USA \\ Received 2009 May 11; accepted 2009 June 29; published 2009 July 13
}

\begin{abstract}
SDSS J091709.55+463821.8 (hereafter J0917+4638) is the lowest surface gravity white dwarf (WD) currently known, with $\log g=5.55 \pm 0.05\left(M \approx 0.17 M_{\odot}\right)$. Such low-mass white dwarfs (LMWDs) are believed to originate in binaries that evolve into $\mathrm{WD} / \mathrm{WD}$ or $\mathrm{WD} /$ neutron star (NS) systems. An optical search for J0917+4638's companion showed that it must be a compact object with a mass $\geqslant 0.28 M_{\odot}$. Here we report on Green Bank Telescope $820 \mathrm{MHz}$ and XMM-Newton X-ray observations of J0917+4638 intended to uncover a potential NS companion to the LMWD. No convincing pulsar signal is detected in our radio data. Our X-ray observation also failed to detect X-ray emission from J0917+4638's companion, while we would have detected any of the millisecond radio pulsars in 47 Tuc. We conclude that the companion is almost certainly another WD.
\end{abstract}

Key words: pulsars: general - stars: individual (SDSS J091709.55+463821.8) - white dwarfs

\section{INTRODUCTION}

Low-mass white dwarfs (LMWDs), generally defined as having $M<0.45 M_{\odot}$, make up a small but highly interesting subset of white dwarfs (WDs). Using the Palomar Green Survey, Liebert et al. (2005) estimated that the formation rate of LMWDs is $0.4 \times 10^{-13} \mathrm{pc}^{-3} \mathrm{yr}^{-1}$, meaning that they make up only $\sim 10 \%$ of the population of the most commonly observed WDs, hydrogen atmosphere DAs. But it is their presumed evolutionary histories that make LMWDs truly intriguing. The youngest WDs in the oldest globular clusters in the Milky Way have masses of $\sim 0.5 M_{\odot}$ (Hansen et al. 2007), implying that lower mass WDs undergo significant mass loss as they form. The preferred scenario is that these WDs form in a tight binary whose evolution includes a phase of mass transfer. As a result, much of the WD progenitor's envelope is removed, preventing a helium flash in its core, and producing a low-mass, helium-core WD.

Brown et al. (2006) identified J0917+4638 as a DA WD in their hyper-velocity star survey of photometrically selected Bstar candidates. Detailed model atmosphere analyses by Kilic et al. (2007a, 2007b) showed that it has $T_{\text {eff }}=11,855 \mathrm{~K}, \log g=$ 5.55 , and $M \approx 0.17 M_{\odot}$ (see Table 1). The lack of evidence of a companion in the optical photometry forces any main-sequence companion to have $M<0.1 M_{\odot}$, ruling out a low-mass main-sequence stellar companion. Radial velocity monitoring uncovered variations with a period of $7.6 \mathrm{hr}$, implying that the mass of the companion is $\geqslant 0.28 M_{\odot}$ (Kilic et al. 2007b).

What is the nature of this companion? While LMWDs are found in WD/WD systems (e.g., Marsh et al. 1995), most known LMWDs are found as companions to neutron stars (NSs), and specifically to NSs "recycled" as millisecond pulsars (MSPs; Panei et al. 2007). Most field radio pulsars in binary systems are MSPs, where a middle-aged, radio-quiet NS has been reactivated as a pulsar via accretion from its

\footnotetext{
8 NSF Astronomy \& Astrophysics Postdoctoral Fellow.

9 Spitzer Fellow.
}

companion. The MSP companions are generally thought to be LMWDs with $M=0.1-0.4 M_{\odot}$, although they are often too faint for optical spectroscopy to confirm that they are LMWDs (see van Kerkwijk et al. 2005). Still, a third of the $\sim 50$ MSP companions discovered outside of globular clusters have $M \lesssim 0.2 M_{\odot}$, assuming the systems have a median inclination of $60^{\circ}$ (Manchester et al. 2005b).

While simulations designed to identify the evolutionary pathways that produce LMWD/MSP systems do not generally predict many systems with $P_{\text {orb }}$ much shorter than a day (e.g., Nelson et al. 2004), and while the system's mass function implies that the probability that J0917+4638 has a WD companion is $89 \%$ (Kilic et al. 2007b), a NS (or black hole) companion to this LMWD cannot be ruled out with the current optical observations. In addition, for the currently known sample of WD/WD systems where both WD masses have been measured, the mass ratio is typically about unity (see Nelemans et al. 2005, and references therein), while the ratio for the J0917+4638 binary system is $\leqslant 0.61$.

Because of the connections between LMWDs and MSPs, we used the Green Bank Telescope (GBT) to search for a putative pulsar companion to J0917+4638, and report here on these observations (Section 2). We also report on an $X M M$-Newton X-ray Observatory observation of this LMWD (Section 3). Blackbody emission from a putative NS companion to the LMWD should be gravitationally bent, and we should detect the NS in X-rays even if it were radio-quiet or if its pulsar beam were missing our line of sight (Beloborodov 2002). We are specifically motivated by the X-ray detection of all known MSPs in the globular cluster 47 Tuc (Heinke et al. 2005; Bogdanov et al. 2006), allowing predictions of the X-ray emission of other MSPs. We choose this sample of MSPs for comparison in part because the distance to globular clusters such as 47 Tuc (4.5 kpc, 2003 update of Harris 1996) are better known than the distances to most MSPs. We discuss the significance of our nondetections and conclude in Section 4. 
Table 1

SDSS J0917+4638: Properties and Observations

\begin{tabular}{ccccccccc}
\hline \hline $\begin{array}{c}\text { SDSS } g \\
(\mathrm{mag})\end{array}$ & $\begin{array}{c}T_{\text {eff }} \\
(\mathrm{K})\end{array}$ & $\begin{array}{c}M_{\mathrm{WD}} \\
\left(M_{\odot}\right)\end{array}$ & $\begin{array}{c}P_{\text {orb }} \\
(\mathrm{hr})\end{array}$ & $\begin{array}{c}\text { Dist. } \\
(\mathrm{kpc})\end{array}$ & $\begin{array}{c}b \\
\left({ }^{\circ}\right)\end{array}$ & $\begin{array}{c}\text { DM } \\
\left(\mathrm{cm}^{-3} \mathrm{pc}\right)\end{array}$ & $\begin{array}{c}\text { GBT } \\
\text { Int. (s) }\end{array}$ & $\begin{array}{c}X M M \\
\text { Int. (s) }\end{array}$ \\
\hline $18.77 \pm 0.02$ & 11855 & $\approx 0.17$ & $7.594 \pm 0.002$ & 2.3 & +44.0 & 80 & 13300 & 23418 \\
\hline
\end{tabular}

Notes. The $g$ (PSF) magnitude is from SDSS Data Release 7 (Abazajian et al. 2009). The distance and orbital period are from Kilic et al. (2007b). The listed DM is the maximum value used when searching for pulsations; it corresponds approximately to twice the maximum value obtained in the direction of J0917+4638 with the Cordes \& Lazio (2002) model.

\section{GREEN BANK TELESCOPE OBSERVATIONS}

J0917+4638 was observed with the GBT on 2007 November 30 . The observing set-up and data reduction were the same as described in Agüeros et al. (2009). At $820 \mathrm{MHz}$, the BerkeleyCaltech Pulsar Machine (Backer et al. 1997) provided $48 \mathrm{MHz}$ of bandwidth split into 96 spectral channels; total power samples for each channel were recorded every $72 \mu \mathrm{s}$. The total observing time was $13,300 \mathrm{~s}(3.7 \mathrm{hr})$. We used standard pulsar search techniques as implemented in the PRESTO software package (Ransom 2001). We calculated the maximum dispersion measure (DM) expected in the direction of J0917+4638 using the Cordes \& Lazio (2002) model for the distribution of free electrons in the Galaxy. To account for uncertainties, we dedispersed the data up to a DM limit twice that obtained from the model, i.e., $\mathrm{DM}=80 \mathrm{~cm}^{-3}$ pc.

No convincing pulsar signal was detected in our data. Below we discuss the limitations of our search.

\subsection{Acceleration Sensitivity}

The orbital motion of a putative pulsar companion to J0917+4638 could significantly affect its apparent spin period. Based on radial velocity monitoring, Kilic et al. (2007b) found that J0917+4638 is in an orbit with a period $7.6 \mathrm{hr}$. Assuming that the LMWD companion is a $1.4 M_{\odot} \mathrm{NS}$, this implies that the maximum orbital acceleration is on the order of $100 \mathrm{~m} \mathrm{~s}^{-2}$, which is significantly larger than what is typically seen in these systems (for $90 \%$ of known pulsars the maximum orbital acceleration is $\leqslant|25| \mathrm{m} \mathrm{s}^{-2}$; Manchester et al. 2005b; van Leeuwen et al. 2007).

Our integration time represents nearly half of the binary orbital period. As a result, the assumption of a constant apparent acceleration built into PRESTO breaks down. We therefore divided our GBT data into 14 separate $900 \mathrm{~s}$ integrations (each representing $\sim 3 \%$ of an orbit) and one $700 \mathrm{~s}$ integration and conducted searches for pulsations separately in each of these partial observations. ${ }^{10}$ This extended our search sensitivity to accelerations on the order of several hundred $\mathrm{m} \mathrm{s}^{-2}$, but as detailed in the following section, reduced our luminosity sensitivity. None of these searches uncovered a convincing pulsar signal.

\subsection{Luminosity Sensitivity}

We use the standard modifications to the radiometer equation to calculate the minimum detectable period-averaged flux density for our searches. We consider a pulsar duty cycle of $20 \%$ (typical of MSPs). At $820 \mathrm{MHz}$, the GBT gain is $2 \mathrm{~K} \mathrm{Jy}^{-1}$ and the system temperature is $25 \mathrm{~K}$. The sky temperature at this

\footnotetext{
${ }_{10}$ We also conducted a search of the entire $3.7 \mathrm{hr}$ integration, which
} unsurprisingly did not return any good candidate pulsar signals. frequency and a Galactic latitude of $b=+44^{\circ}$ only adds a few $\mathrm{K}$ to the overall temperature. We consider an effective threshold signal-to-noise ratio of 10 . For $t_{\text {int }}=900 \mathrm{~s}$, the sensitivity limit for a long period pulsar at the beam center is $\sim 0.26 \mathrm{mJy}$.

Pulsar luminosities are often measured at $1400 \mathrm{MHz}$; using a typical spectral index of -1.7 , the limiting sensitivity at that frequency is $S_{1400} \approx 0.10 \mathrm{mJy}$ when searching the $900 \mathrm{~s}$ integrations. For an MSP period of $3 \mathrm{~ms}$, our sensitivity at $1400 \mathrm{MHz}$ was roughly $0.14 \mathrm{mJy}$ for each integration, and it quickly degraded for shorter periods; it was $10 \times$ worse for $1 \mathrm{~ms}$.

The distance to J0917+4638 is estimated to be $2.3 \mathrm{kpc}$ (Kilic et al. 2007b), implying that our $L_{1400} \equiv S_{1400} d^{2}$ limits for 3 ms periods are $\approx 0.7 \mathrm{mJy} \mathrm{kpc}^{2}$ for each $900 \mathrm{~s}$ integration. According to the ATNF's pulsar catalog ${ }^{11}$ (Manchester et al. 2005a), of the 50 MSPs (periods $<25 \mathrm{~ms}$ ) outside of globular clusters and with measured luminosities, $64 \%$ have $L_{1400}>$ $0.7 \mathrm{mJy} \mathrm{kpc}{ }^{2}$. We would therefore expect our search to detect roughly two-thirds of the known MSPs were one orbiting J0917+4638 and beaming radio waves toward the Earth.

We note that J0917+4638 falls within the FIRST footprint and is not detected in that $1.4 \mathrm{GHz}$ survey, for which the sensitivity limit is roughly $1 \mathrm{mJy}$ (Becker et al. 1995).

\section{XMM-NEWTON OBSERVATION}

\subsection{Motivation}

MSP radio beaming fractions are $<100 \%$, and as a result, some MSPs have not yet been detected in the radio in binary systems where there is strong evidence for their presence (e.g., the companion to the young pulsar PSR J1906+0746; Lorimer et al. 2006). Given that the NS blackbody emission is gravitationally bent, allowing us to view $>75 \%$ of the NS surfaces in X rays (Beloborodov 2002), sufficiently deep X-ray observations are virtually guaranteed to detect these MSPs.

Heinke et al. (2005) found no correlation between the $\mathrm{X}$-ray and radio luminosities of MSPs in 47 Tuc, as expected due to the differing nature and spatial location of the X-ray and radio emission, and found that all MSPs in $47 \mathrm{Tuc}^{12}$ have Xray luminosities ranging between $L_{X}(0.5-6 \mathrm{keV})=2 \times 10^{30}$ and $2 \times 10^{31} \mathrm{erg} \mathrm{s}^{-1}$. Bogdanov et al. (2006) showed that the X-ray spectra of the MSPs in 47 Tuc are typically dominated by thermal blackbody-like emission from the NS surface around the polar caps, with temperature $1-3 \times 10^{6} \mathrm{~K}$. This X-ray emission is sometimes overwhelmed by additional nonthermal $\mathrm{X}$ rays

\footnotetext{
${ }_{11}$ http://www.atnf.csiro.au/research/pulsar/psrcat/.

12 Only 15 MSPs in 47 Tuc have published locations farther than $1^{\prime \prime}$ from other MSPs; two pairs of MSPs that are closer cannot be conclusively resolved (Bogdanov et al. 2006). However, the flux from each pair is consistent with expectations from the other MSPs.
} 
that are either magnetospheric or due to an intra-binary shock. Bogdanov et al. (2006) also showed that there are no clear systematic differences between the X-ray properties of MSPs in 47 Tuc and in the Galactic field. Thus, we requested an XMM observation capable of detecting any of the known MSPs in 47 Tuc, were they located at the distance of J0917+4638.

J0917+4638 had not previously been observed in the X-ray since the ROSAT All-Sky Survey (Voges et al. 1999, 2000), where it was not detected (unsurprisingly, considering the short exposure time).

\subsection{X-Ray Data Analysis}

We observed J0917+4638 on 2008 May 7 for 17 ks (ObsID 0553440101) with XMM's EPIC camera, consisting of two MOS CCD detectors (Turner et al. 2001) and a pn CCD detector (Strüder et al. 2001). All data were reduced using FTOOLS ${ }^{13}$ and SAS version 8.0.0. ${ }^{14}$ We excluded times of soft proton background flaring, when the pn camera's count rate exceeded $25(0.2-10 \mathrm{keV})$ counts $\mathrm{s}^{-1}$, or when the MOS cameras exceeded 7 or $8(0.2-10 \mathrm{keV})$ counts s$^{-1}$ for the MOS1 or MOS2 cameras respectively. This left $8.9 \mathrm{ks}$ of good data from the pn detectors, and $11.3 \mathrm{ks}$ from the MOS detectors. We filtered the events on pixel patterns (trying PATTERN $<=1$ or $<=4$ for pn and PATTERN $<=12$ for MOS data), and for $\mathrm{FLAG}==0$. We choose an energy range of $0.2-1.5 \mathrm{keV}$ to obtain optimal sensitivity to the soft blackbody emission expected from MSPs.

No source is detected at or within $1^{\prime}$ of the location of J0917+4638 either with detection algorithms or by eye. We utilize our knowledge of the $X M M$ point spread function ${ }^{15}$ and absolute pointing accuracy $\left(<1^{\prime \prime}\right.$; Kirsch et al. 2004) to determine an upper limit. For the pn, $50 \%$ of $1.5 \mathrm{keV}$ photons are found within $8^{\prime \prime}$, and $80 \%$ within $20^{\prime \prime}$. For the MOS cameras, $50 \%$ of $1.5 \mathrm{keV}$ photons are recorded within 8 , and $75 \%$ within $20^{\prime \prime}$. We find 3 counts within an $8^{\prime \prime}$ circle, or 20 counts within a $20^{\prime \prime}$ circle, in the combined image. This is consistent with a nondetection, as the expected background counts in these circles are $3.2 \pm 0.2$ and $19.8 \pm 1.3$ counts, respectively, as derived from nearby background regions.

\subsection{Comparison to MSPs in 47 Tuc}

We use the X-ray faintest MSP in 47 Tuc, 47 Tuc T, to calibrate our expectations for the detection of an MSP in J0917+4638. 47 Tuc T has $L_{X}(0.2-1.5 \mathrm{keV})=1.5 \times 10^{30} \mathrm{erg} \mathrm{s}^{-1}$ and a $134 \mathrm{eV}$ blackbody spectrum (Bogdanov et al. 2006). We use PIMMS ${ }^{16}$ to determine the expected EPIC count rates from 47 Tuc $\mathrm{T}$ were it located at $2.3 \mathrm{kpc}$ (the distance to J0917+4638) behind an estimated $N_{H}=1.5 \times 10^{20} \mathrm{~cm}^{-2}$ (Dickey \& Lockman 1990). We expect 10.9 counts within $8^{\prime \prime}$, or 17.0 within $20^{\prime \prime}$, accounting for the relevant encircled energy fractions, from such an MSP. Comparing the predicted counts with the Poisson errors on the detected counts (Gehrels 1986, Equation (7)), we find that the number of counts within $20^{\prime \prime}$ is $3.0 \sigma$ below expectations for the faintest known MSP in 47 Tuc, while the counts within $8^{\prime \prime}$ are $3.5 \sigma$ below those expectations. 47 Tuc $\mathrm{T}$ is the $\mathrm{X}$-ray

\footnotetext{
$13 \mathrm{http} / / /$ heasarc.gsfc.nasa.gov/docs/software/ftools/ftools_menu.html

$14 \mathrm{http}: / / \mathrm{xmm}$.vilspa.esa.es

15 XMM-Newton User's Handbook,

http://xmm.esac.esa.int/external/xmm_user_support/

documentation/uhb/index.html

$16 \mathrm{http}: / /$ asc.harvard.edu/toolkit/pimms.jsp
}

faintest of the 15 independently measured MSPs in 47 Tuc; the median X-ray luminosity is $2.1 \times$ greater (Bogdanov et al. 2006 ), which is ruled out at $6.3 \sigma$ confidence. Our nondetection is therefore strong evidence against the existence of an MSP in $\mathrm{J} 0917+4638$.

\section{CONCLUSION}

We have searched for evidence of an MSP companion to the LMWD J0917+4638 through radio and X-ray observations. Our radio search reaches a sensitivity sufficient to detect roughly two-thirds of the known MSPs, while our X-ray search is sensitive enough to detect any of the 15 independently identified MSPs in 47 Tuc. Together, our nondetections provide strong evidence against the presence of an MSP in this system. Furthermore, since any NS companion to J0917+4638 would presumably have been recycled through accretion from the LMWD, we rule out the presence of a NS in this system. Although a black hole companion is still conceivable (as the $7.6 \mathrm{hr}$ orbital period would not induce current accretion and Xray activity), such a companion is far less probable than a WD companion given both the system's mass function (Kilic et al. 2007b) and the stellar initial mass function for $M \geqslant 1 M_{\odot}$ (e.g., Scalo 1998). We conclude that J0917+4638's more massive companion $\left(M \geqslant 0.28 M_{\odot}\right)$ is almost certainly another WD.

Roughly two dozen WD/WD binaries are known and in ten such systems both WD masses have been measured (see Nelemans et al. 2005, and references therein). The individual masses of WDs in these systems range between 0.29 and $0.71 M_{\odot}$; the median mass for those with measured masses (and not just lower limits) is $0.43 M_{\odot}$. The majority of these double WD systems have mass ratios near unity, which is contrary to what is expected from standard population synthesis models (Nelemans \& Tout 2005). This has been used to argue that energy balance ( $\alpha$-formalism), the standard prescription for common envelope evolution, should be replaced by angular momentum balance ( $\gamma$-algorithm; Maxted et al. 2002; Nelemans et al. 2005).

In particular, Nelemans et al. (2005) found that the $\alpha$ formalism cannot be used to describe the first phase of mass transfer for nine of the ten double WD systems in which both WD masses have been measured. The exception is WD1704+481, which has a mass ratio $=0.7$, similar to the expected ratio from the $\alpha$-formalism. The mass ratio for the J0917+4638 binary system is $\leqslant 0.61$. Recent observations of another LMWD, LP400-22, showed that it is in a binary with a mass ratio $\leqslant 0.46$ (Kilic et al. 2009). The mass ratios of these systems imply that the $\alpha$-formalism may apply to the evolutionary history of some of the WD/WD binaries. Determining the mass ratios of the other SDSS LMWD systems for which the nature of the companion is unknown will be important in understanding the role of energy versus angular momentum balance in reconstructing common envelop evolution.

M.A.A. is supported by an NSF Astronomy and Astrophysics Postdoctoral Fellowship under award AST-0602099. C.H. is supported by NSERC. Further support was provided to M.K. through the Spitzer Space Telescope Fellowship Program, through a contract issued by the JPL/Caltech under a contract with NASA.

The Robert C. Byrd Green Bank Telescope is operated by the National Radio Astronomy Observatory, which is a facility of the US National Science Foundation operated under cooperative 
agreement by Associated Universities, Inc. XMM-Newton is an ESA science mission with instruments and contributions directly funded by ESA Member States and NASA.

\section{REFERENCES}

Abazajian, K., et al. 2009, ApJS, 182, 543

Agüeros, M. A., et al. 2009, ApJ, 697, 283

Backer, D. C., et al. 1997, PASP, 109, 61

Becker, R. H., et al. 1995, ApJ, 450, 559

Beloborodov, A. M. 2002, ApJ, 566, L85

Bogdanov, S., Grindlay, J. E., Heinke, C. O., Camilo, F., Freire, P. C. C., \& Becker, W. 2006, ApJ, 646, 1104

Brown, W. R., et al. 2006, ApJ, 647, 303

Cordes, J. M., \& Lazio, T. J. W. 2002, arXiv:astro-ph/0207156

Dickey, J. M., \& Lockman, F. J. 1990, ARA\&A, 28, 215

Gehrels, N. 1986, ApJ, 303, 336

Hansen, B. M. S., et al. 2007, ApJ, 671, 380

Harris, W. E. 1996, VizieR Online Data Catalog, 7195, 0

Heinke, C. O., Grindlay, J. E., Edmonds, P. D., Cohn, H. N., Lugger, P. M., Camilo, F., Bogdanov, S., \& Freire, P. C. 2005, ApJ, 625, 796

Kilic, M., Allende Prieto, C., Brown, W. R., \& Koester, D. 2007a, ApJ, 660, 1451

Kilic, M., Brown, W. R., Allende Prieto, C., Pinsonneault, M. H., \& Kenyon, S. J. 2007b, ApJ, 664, 1088
Kilic, M., Brown, W. R., Prieto, C. A., Swift, B., Kenyon, S. J., Liebert, J., \& Agüeros, M. A. 2009, ApJ, 695, L92

Kirsch, M. G. F., et al. 2004, Proc. SPIE 5488, 103

Liebert, J., et al. 2005, ApJS, 156, 47

Lorimer, D. R., et al. 2006, ApJ, 640, 428

Manchester, R. N., Hobbs, G. B., Teoh, A., \& Hobbs, M. 2005a, VizieR Online Data Catalog, 7245, 0

Manchester, R. N., Hobbs, G. B., Teoh, A., \& Hobbs, M. 2005b, AJ, 129, 1993

Marsh, T. R., et al. 1995, MNRAS, 275, 828

Maxted, P. F. L., Marsh, T. R., \& Moran, C. K. J. 2002, MNRAS, 332, 745

Nelemans, G., \& Tout, C. A. 2005, MNRAS, 356, 753

Nelemans, G., et al. 2005, A\&A, 440, 1087

Nelson, L. A., Dubeau, E., \& MacCannell, K. A. 2004, ApJ, 616, 1124

Panei, J. A., Althaus, L. G., Chen, X., \& Han, Z. 2007, MNRAS, 382, 779

Ransom, S. M. 2001, PhD thesis, Harvard Univ.

Scalo, J. 1998, in ASP Conf. Ser. 142, The Stellar Initial Mass Function: 38th Herstmonceux Conference, ed. G. Gilmore \& D. Howell (San Francisco, CA: ASP), 201

Strüder, L., et al. 2001, A\&A, 365, L18

Turner, M. J. L., et al. 2001, A\&A, 365, L27

van Kerkwijk, M. H., et al. 2005, in ASP Conf. Ser. 328, Binary Radio Pulsars, ed. F. A. Rasio \& I. H. Stairs (San Francisco, CA: ASP), 357

van Leeuwen, J., et al. 2007, MNRAS, 374, 1437

Voges, W., et al. 1999, A\&A, 349, 389

Voges, W., et al. 2000, VizieR Online Data Catalog, 9029, 0 